\title{
Similarity solutions for the self-dual SU(2) fields
}

\author{
M. Halilsoy \\ Nuclear Engineering Department, King Abdulaziz University, P. O. Box 9027, Jeddah 21413, Saudi Arabia
}

(Received 20 December 1985)

\begin{abstract}
We present new solutions to Yang's self-dual SU(2) equations. These solutions have the property that they are self-similar, together with some of their elliptical and transcendental extensions.
\end{abstract}

\section{INTRODUCTION}

Since the publication of Yang's original work ${ }^{1}$ on the self-dual SU(2) gauge theory, much effort has been put forward to integrate these equations. ${ }^{2}$ Although this work was carried out in the four-dimensional Euclidean space-in connection with instantons-there is always freedom to suppress two of the coordinates and study the theory in a plane. Such a reduced formalism turns out to have much in common with Einstein's equations that admit two Killing vectors, a topic to which much access has been attained in the relativity community. ${ }^{3}$ By the same token, we integrate Yang's equations once more in analogy with the similarity integral that we had obtained previously in the EinsteinMaxwell theory. ${ }^{4}$

Static, axially symmetric self-dual Yang's equations in the $R$ gauge can be derived by the variation of the action

$$
E[\phi, \Psi, \bar{\Psi}]=\int \frac{|\nabla \Psi|^{2}+(\nabla \phi)^{2}}{\phi^{2}} \rho d \rho d z
$$

where the real function $\phi$ and the complex function $\Psi$ depend only on $\rho$ and $z$. This action is in the form of an energy functional of the harmonic maps, $f: M \rightarrow M^{\prime}$, where the respective manifolds are

$$
\begin{aligned}
& M: d s^{2}=d \rho^{2}+d z^{2}+\rho^{2} d \varphi^{2}, \\
& M^{\prime}: d s^{2}=\frac{|d \Psi|^{2}+d \phi^{2}}{\phi^{2}} .
\end{aligned}
$$

Our purpose is to consider composite maps, where a third manifold $M^{\prime \prime}$ is introduced between the manifolds $M$ and $M^{\prime}$. By a corollary, ${ }^{5}$ the composition of a geodesic map and a harmonic map itself is harmonic; henceforth we consider the harmonic maps from $M^{\prime \prime}$ into $M^{\prime}$, where

$$
M^{\prime \prime}: d s^{\prime \prime 2}=d v^{2}
$$

is a one-dimensional manifold. The requirement that the maps from $M$ into $M^{\prime \prime}$ are harmonic restricts $v$ to be the arbitrary harmonic function in the $\rho, z$ coordinates, i.e.,

$$
v_{\rho \rho}+\frac{1}{\rho} v_{\rho}+v_{z z}=0
$$

The energy functional of the maps from $M^{\prime \prime}$ into $M^{\prime}$ reads

$$
E[\phi, \Psi, \bar{\Psi}]=\int d v \frac{\left|\Psi^{\prime}\right|^{2}+\phi^{\prime 2}}{\phi^{2}},
$$

where prime denotes $d / d v$, so that Yang's equations reduce to

$$
\begin{aligned}
& \phi \phi^{\prime \prime}-\phi^{\prime 2}+\left|\Psi^{\prime}\right|^{2}=0, \\
& \phi \Psi^{\prime \prime}-2 \phi^{\prime} \Psi^{\prime}=0, \\
& \phi \bar{\Psi}^{\prime \prime}-2 \phi^{\prime} \bar{\Psi}^{\prime}=0 .
\end{aligned}
$$

The integration of these equations is in the sequel.

\section{COMPLETE SIMILARITY INTEGRAL}

One notices first that Eqs. (6)-(8) are equivalent to

$$
\Psi^{\prime}=m_{0} \phi^{2}
$$

and

$$
\phi \phi^{\prime \prime}-\phi^{\prime 2}+\left|m_{0}\right|^{2} \phi^{4}=0,
$$

where $m_{0}$ is a complex integration constant. Equation (10) is the typical Liouville equation for the function $\ln \phi$, which admits the solution

$$
\phi=\frac{1}{\cosh \left(\left|m_{0}\right| v\right)},
$$

and, therefore,

$$
\Psi=\frac{m_{0}}{\left|m_{0}\right|} \tanh \left(\left|m_{0}\right| v\right)+n_{0}
$$

with $n_{0}$ another constant. We note that since a constant is harmonic, we have the freedom to omit an additive constant to the variable $v$.

The foregoing solution can be obtained alternatively by making use of the Hamilton-Jacobi ( $\mathrm{HJ}$ ) theory. The $\mathrm{HJ}$ functional is to be parametrized by $v$ and the $\mathrm{HJ}$ equation reads

$$
\frac{\partial S}{\partial v}+H\left(\Psi, \phi, \frac{\partial S}{\partial \Psi}, \frac{\partial S}{\partial \phi}\right)=0,
$$

where the Hamiltonian is defined by

$$
H=|g|^{-1 / 2} g^{\prime A B} \frac{\partial S}{\partial f^{A}} \frac{\partial S}{\partial f^{B}},
$$

and where $f^{A}$ are the coordinates of the $M^{\prime}$ metric. Choosing $\Psi=\chi e^{i \lambda}$, the $\mathrm{HJ}$ equation becomes

$$
\phi^{-2} \frac{\partial S}{\partial v}+\left(\frac{\partial S}{\partial \chi}\right)^{2}+\left(\frac{\partial S}{\partial \phi}\right)^{2}+\frac{1}{\chi^{2}}\left(\frac{\partial S}{\partial \lambda}\right)^{2}=0
$$


whose separable solution can be expressed by

$S=-a_{1} v+a_{3} \lambda+\int^{x}\left(a_{2}-\frac{a_{3}^{2}}{\chi^{2}}\right)^{1 / 2} d x+\int\left(\frac{a_{1}^{2}}{\phi^{2}}-a_{2}\right)^{1 / 2} d \phi$.

Here $a_{1}, a_{2}$, and $a_{3}$ are nontrivial constants and the selfdual similarity solution sought reduces then to the equations

$$
\frac{\partial S}{\partial a_{1}}=0, \quad \frac{\partial S}{\partial a_{2}}=0, \quad \frac{\partial S}{\partial a_{3}}=0 \text {. }
$$

Although this solution is a rather simple one, it has the feature that its independent variable occurs as an arbitrary harmonic function.

\section{AN ELLIPTICAL SOLUTION}

We reparametrize the foregoing functions by

$$
\begin{aligned}
& \phi=y \cos \Omega, \\
& \Omega=y \sin \Omega e^{i \lambda+i b \tilde{v}} \quad(b \text { is a real constant }),
\end{aligned}
$$

and consider the harmonic map between

$$
M^{\prime \prime}: d s^{\prime \prime 2}=d v^{2}+d \tilde{v}^{2}
$$

and

$$
M^{\prime}: d s^{\prime 2}=\frac{|\Psi|^{2}+d \phi^{2}}{\phi^{2}} .
$$

As is observed, we make $M^{\prime \prime}$ a two-dimensional manifold, where $\tilde{v}$ is a new function whose Jacobian with $v$ must not vanish everywhere. The functions $y, \Psi$, and $\lambda$ are still only functions of $v$. The energy functional constructed from $M^{\prime \prime}$ into $M^{\prime}$ will yield the Lagrangian

$$
L=\left(\frac{y^{\prime}}{y \cos \Omega}\right)^{2}+\left(\frac{\Omega^{\prime}}{\cos \Omega}\right)^{2}+\tan ^{2} \Omega\left(\lambda^{\prime 2}+b^{2}\right) .
$$

The Yang equations resulting from the variational principles admit the first integrals

$$
\begin{aligned}
& \tan ^{2} \Omega \lambda^{\prime}=c_{0}, \\
& y^{\prime}=a_{0} y \cos ^{2} \Omega,
\end{aligned}
$$

where $c_{0}$ and $a_{0}$ are both real integration constants. The equation for $\Omega$ turns out to be nontrivial:

$$
\Omega^{\prime \prime}-\sin \Omega \cos ^{3} \Omega a_{0}^{2}+\tan \Omega\left(\Omega^{\prime 2}-c_{0}^{2} \cot ^{2} \Omega-b^{2}\right)=0 .
$$

Defining a new function by $M=\operatorname{arctanh}(\sin \Omega)$, this equation is transformed into

$$
M^{\prime \prime}-a_{0}^{2} \frac{\sinh M}{\cosh ^{3} M}-c_{0}^{2} \frac{\cosh M}{\sinh ^{3} M}-b^{2} \sinh M \cosh M=0,
$$

which is equivalent to the expression

$$
\int \frac{d R}{\left[b^{2} R^{3}+\left(b^{2}+l\right) R^{2}+\left(l-a_{0}^{2}-c_{0}^{2}\right) R-c_{0}^{2}\right]^{1 / 2}}=2 v .
$$

Note that we have redefined $R=\sinh ^{2} M$ and $l$ is a new constant of integration. It is known that for $b \neq 0$ this can be transformed into the standard elliptical forms by the proper choice of the constants $A$ and $B$ in the transformation ${ }^{6}$ $R=(A+B x) /(1+x)$; however, we shall not pursue it further here. Assuming this has been carried out, the final solution is

$$
\lambda=\text { const }+c_{0} \int \frac{d v}{R}
$$

and

$$
y=\text { const } \exp \left(a_{0} \int \frac{d v}{1+R}\right) .
$$

\section{A TRANSCENDENT SOLUTION}

As a final class of solutions we show that the self-dual Yang-Mills equations admit solutions expressible in terms of Painleve's fifth transcendents. Although this class was discovered before, ${ }^{7}$ we shall rederive it by an alternative method.

We choose the $M^{\prime \prime}$ manifold to be in one of the following forms:

(i) $d s^{\prime \prime 2}=e^{2 v} d v^{2}+d \tilde{v}^{2}+e^{2 v} d \varphi^{2}$,

(ii) $d s^{\prime \prime 2}=d v^{2}+d \tilde{v}^{2} e^{-v}+e^{v} d \varphi^{2}$,

and $\Psi$ is chosen as in Sec. III, $\Psi=y \sin \Omega e^{i(\lambda+\beta \tilde{v})}$, where $\beta$ is a real constant. The Lagrangian of the new map takes the form

$$
L=\left(\frac{y^{\prime}}{y \cos \Omega}\right)^{2}+\left(\frac{\Omega^{\prime}}{\cos \Omega}\right)^{2}+\tan ^{2} \Omega\left(\lambda^{\prime 2}+\beta^{2} e^{2 v}\right),
$$

and the $\Omega$ equation is modified to

$$
\begin{aligned}
\frac{d^{2} w}{d v^{2}}-\left(\frac{d w}{d v}\right)^{2} & \left(\frac{1}{2 w}+\frac{1}{w-1}\right) \\
& -2(1-w)^{2}\left(a_{0}^{2} w+\frac{c_{0}^{2}}{w}\right)-2 \beta^{2} e^{2 v} w=0
\end{aligned}
$$

where $w=\sin ^{2} \Omega$. Changing the independent variable by $x=e^{2 v}$, this equation becomes

$$
\begin{aligned}
w_{x x}+\frac{1}{x} w_{x}-w_{x}{ }^{2}\left(\frac{1}{2 w}\right. & \left.+\frac{1}{w-1}\right) \\
& -\frac{(1-w)^{2}}{2 x^{2}}\left(a_{0}^{2} w+\frac{c_{0}^{2}}{w}\right)-\frac{\beta^{2}}{2 x} w=0,
\end{aligned}
$$

which is a particular Painleve's fifth transcendent, whose general form is

$$
\begin{aligned}
& w_{x x}+\frac{1}{x} w_{x}-w_{x}^{2}\left(\frac{1}{2 w}+\frac{1}{w-1}\right) \\
& +\frac{(1-w)^{2}}{x^{2}}\left(\alpha w+\frac{\epsilon}{w}\right)+\frac{\gamma}{x} w+\delta w \frac{w+1}{w-1}=0 .
\end{aligned}
$$

It is crucial that either $\delta \neq 0$ (as in Einstein-Maxwell theory ${ }^{8}$ ) or $\gamma \neq 0$ (in this case) in order that the transcendental nature remains. Let us note however that we have yet to meet the case where both $\gamma \neq 0$ and $\delta \neq 0$. The final 
solution is

$$
\lambda=\mathrm{const}+\int\left(w^{-1}-1\right) d v, \quad \phi=\mathrm{const}(1-w)^{1 / 2} \exp \left(a_{0} \int(1-w) d v\right), \quad e^{-i \lambda} \Psi=\frac{w^{1 / 2}}{(1-w)^{1 / 2} \phi e^{i \beta \tilde{v}}},
$$

where $v$ is harmonic and $\tilde{v}$ is an arbitrary function. We must add, however, that once we want to recover axial symmetry in the problem, we are bound to make the choices $v=\ln \rho$ and $\tilde{v}=z$ for the base manifold (26), which will result in the particular solution already given in Ref. 7 .

${ }^{1}$ C. N. Yang, Phys. Rev. Lett. 38, 1377 (1977).

2B. C. Xanthopoulos, J. Phys. A 14, 1445 (1981).

${ }^{3}$ L. Witten, Phys. Rev. D 19, 718 (1979).

${ }^{4} \mathrm{M}$. Halilsoy, Lett. Nuovo Cimento 37, 231 (1983).

${ }^{5}$ J. Eells and J. H. Samson, Am. J. Math. 86, 109 (1964).
6J. Edwards, Treatise on Integral Calculus (Chelsea, New York, 1922), Vol. II, p. 581.

${ }^{7}$ B. Leaute and G. Marcilhacy, Phys. Lett. 93A, 394 (1983).

${ }^{8} \mathrm{M}$. Halilsoy, Lett. Nuovo Cimento 44, 88 (1985). 